\title{
Schilddrüsenkarzinom
}

\section{„Fast-Track“-Konzept mit rekombinantem humanem TSH}

Durch den Einsatz von rekombinantem humanem Thyreoidea-stimulierendem Hormon (rhTSH) im Rahmen eines „Fast-Track“-Konzepts kann die Behandlung des differenzierten Schilddrüsenkarzinoms (DTC) innerhalb eines 10-tägigen stationären Aufenthalts abgeschlossen werden.

Die DTC-Standardtherapie besteht in einer totalen Thyreoidektomie, der sich die Radioiodablation des Restschilddrüsengewebes anschließt, sobald für die ${ }^{131}$ lod-Aufnahme optimale TSH-Spiegel (>30mU/l) vorliegen. Um einen ausreichenden TSH-Anstieg zu erreichen, wird nach dem herkömmlichen Verfahren die Thyroxin-Einnahme um bis zu 5 Wochen verschoben. Die Kehrseite besteht laut Prof. Dr. Georg Scheumann, Han- nover, in einer Hypothyreose, die erhebliche Beschwerden bereiten und die Wundheilung beeinträchtigen kann. Zudem lehnen es viele Patienten ab, dass sie 4 bis 5 Wochen nach der Operation erneut in die Klinik müssen und in der Zwischenzeit aufgrund von Hypothyreose-Beschwerden eingeschränkt arbeitsfähig sind.

\section{Randomisierte Studie bestätigt Vorteile des „Fast-Track“-Konzepts}

Scheumann und Mitarbeiter bieten ihren Patienten ein „Fast-Track"-Konzept an, das auf der Stimulation der ${ }^{131}$ lod-Aufnahme durch rhTSH (Thyrogen ${ }^{\oplus}$ ) basiert: An Tag 2 nach stationärer Aufnahme erfolgt die Operation, danach die rhTSH-Gabe (Tag 5+6) und die Radioiodablation (Tag 7) sowie nach insgesamt 9 bis 10 Tagen die Entlassung aus der Klinik.

In einer randomisierten Studie mit 25 Patienten wurde das „Fast-Track"-Konzept mit dem herkömmlichen Verfahren verglichen (Emmanouilidis $\mathrm{N}$ et al., 2009, Eur J Endocrinol 161: 763-769). In der rhTSHGruppe war das Intervall zwischen Operation und Entlassung nach Radioablation im Vergleich zum herkömmlichen Verfahren signifikant kürzer (10,1 Tage versus 36,2 Tage; $p<0,0001)$. Die Patienten der rhTSHGruppe waren verglichen mit Patienten, die nach herkömmlichem Verfahren behandelt wurden, an signifikant weniger Tagen arbeitsunfähig (5,9 Tage versus 28,8 Tage; $\mathrm{p}<0,0001)$. GS

Quelle: Veranstaltung der Genzyme $\mathrm{GmbH}$

\section{Follikuläres Lymphom}

\section{Behandlungsstandard Rituximab-Erhaltungstherapie}

Noch in den 1990er-Jahren war aus den Studiendaten zur Therapie des follikulären Lymphoms abzuleiten, dass die Wahl und der Erfolg der Chemotherapie keinen Einfluss auf den Verlauf der Erkrankung haben, und somit die Chemotherapie den Krankheitsverlauf nicht verändern kann. Heute muss man laut Dr. Ingo Schwaner, Berlin, sagen: Rituximab plus Chemotherapie ändert den Verlauf der Erkrankung, Rituximab hat einen wesentlichen Einfluss auf den Verlauf der Erkrankung unabhängig vom gewählten Chemotherapieprotokoll, und die Remissionstiefe von Rituximab plus Chemotherapie hat einen wesentlichen Einfluss auf den Verlauf der Erkrankung.

Eine verbesserte Prognose durch die Behandlung mit Rituximab plus Chemotherapie sowie anschließender Rituximab-Erhaltungstherapie ist in der aktuellen ESMO-Leitlinie zur Diagnose und Behandlung des follikulären Lymphoms nicht mehr nur für die Rezidiv-, sondern auch für die First-line-Situation festgehalten (Dreyling Met al., 2010, Ann Oncol 21: v181-183). Diese Aktualisierung basiert auf den Daten der Phase-III-Studie PRIMA (Primary Rituximab and Maintenance; Salles GA et al., 2010, ASCO: \#4008, oral presentation; Salles G et al., 2011, Lancet 377: 42-51). In dieser Studie erhielten Patienten mit follikulärem Lymphom, die auf first-line Rituximab plus Chemotherapie angesprochen hatten, randomisiert für 2 Jahre eine Erhaltungstherapie mit Rituximab $\left(375 \mathrm{mg} / \mathrm{m}^{2} \mathrm{KOF}\right.$ alle 8 Wochen; $\mathrm{n}=505)$ oder wurden für 2 Jahre beobachtet $(\mathrm{n}=513)$. Im primären Endpunkt progressionsfreies Überleben (PFS) seit der Randomisierung zeigen die aktualisierten 3-Jahres-Daten - wie bereits die erste Analyse nach median 2 Jahren Follow-up - einen signifikanten Vorteil zugunsten der Rituximab-Erhaltungstherapie (Abb.). Wäh-

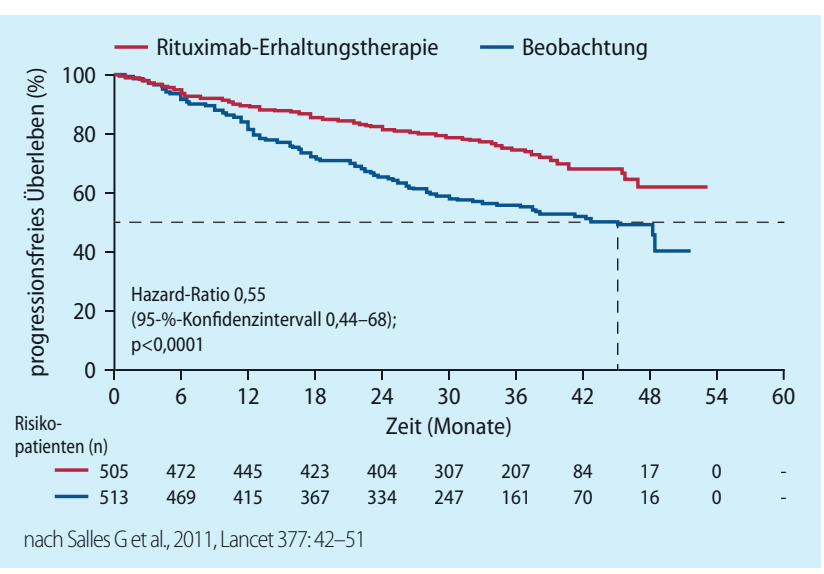

PRIMA-Studie: progressionsfreies Überleben seit der Randomisierung

rend das mediane PFS unter alleiniger Beobachtung laut Schwaner bei ca. 45 Monaten lag, war der Median unter der Rituximab-Erhaltungstherapie noch nicht erreicht. Dabei profitierten die Patienten von der Erhaltungstherapie unabhängig von Alter, FLIPI (Follicular Lymphoma International Prognostic Index) und Ansprechen auf die Induktionstherapie (partielles bzw. komplettes Ansprechen). Interessant bezüglich des Ansprechens auf die Erhaltungstherapie mit Rituximab ist laut Schwaner die Konversion der partiellen Remission in die komplette Remission, da „wir wissen, dass die Patienten, die eine komplette Remission erreichen, länger überleben“. Die Konversionsrate betrug unter der Erhaltungstherapie 52\% versus 30\% bei alleiniger Beobachtung. $A M$

Quelle: Veranstaltung der Roche Pharma AG 